\title{
Characterization of buried heat sources using Dirac excitation
}

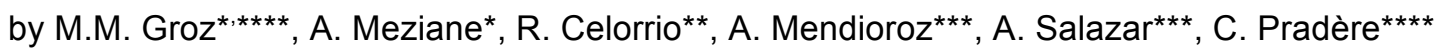 \\ * Université de Bordeaux, CNRS, I2M Bordeaux, 351 cours de la Libération, F-33405 Talence, France, \\ anissa.meziane@u-bordeaux.fr \\ ** Departamento de Matemática Aplicada, EINA/IUMA, Universidad de Zaragoza, Campus Rio Ebro, Edificio \\ Torres Quevedo, 50018 Zaragoza, Spain. \\ *** Departamento de Física Aplicada I, Escuela de Ingeniería de Bilbao, Universidad del País Vasco UPV/EHU, \\ Plaza Ingeniero Torres Quevedo 1, 48013 Bilbao, Spain. \\ **** I2M, UMR CNRS 5295, esplanade des arts et métiers, F-33405 Talence, France christophe.pradere@u- \\ bordeaux.fr
}

\section{Abstract}

A method for characterizing buried heat Dirac sources is proposed in this work. The direct problem is first presented with the analytical solution of the temperature distribution as well as the artifice used to discretize and linearize the problem. Then, the inverse problem is presented with the first results obtained, especially the importance of the time parameter, and the possibility to distinguish two clustered sources.

\section{Introduction}

Infrared (IR) thermography enables to measure the surface temperature fields of a sample. Then, for several problems where volumetric energy conversion (thermoacoustic, thermomechanic, thermoinductive...) occurred, it is very difficult to retrieve the location, the shape and the amplitude of such heat source just from surface temperature data. Recently, a first method has been developed in [1-3], based on inverse algorithm with different kinds of excitations (lockin, burst) and with different shapes of planar heat sources (rectangles, triangles, semicircles...).

In this paper, the problem of a Dirac heat source (spatial and temporal) is studied. It is a realistic model of the heat generation and propagation from small inclusions excited by ultrasonic or inductive means and detected by an IR camera. Figure 1 illustrates the problem with a shape temperature distribution that can be obtained with 3 points-like heat sources. The main goal is to develop a robust and stable inversion algorithm to reconstruct those point-like heat sources and to analyze the possible advantages with respect to other approaches.

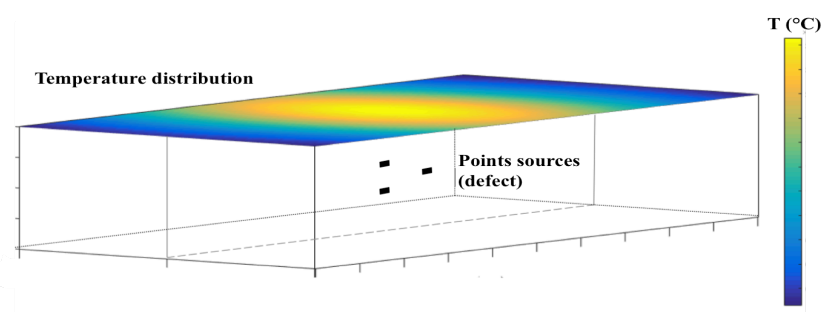

Fig. 1. Shape of temperature distribution obtained with 3 points-like heat sources in the sample

Furthermore, this method could be used as an alternative of the deconvolution of the point spread function in the domain of microscopic image processing.

\section{Characterization of Dirac heat sources by using impulse thermal response}

The method of characterization is based on inverse algorithm and therefore requires the analytical solution of the "direct problem".

\subsection{Direct problem: analytical solution}

The impulse thermal response of a unit instantaneous $(t=0)$ point heat source located at $\left(x^{\prime}, y^{\prime}, z^{\prime}\right)$ in an isotropic material is given by Eq. (1) where the coefficients $\alpha, \rho, c_{p}$ correspond to thermal diffusivity, density and specific heat, respectively. 


$$
T_{\text {Dirac }}(x, y, z, t)=\frac{1}{8 \rho c_{p}(\pi \alpha t)^{3 / 2}} \exp \left(\frac{\left(x-x^{\prime}\right)^{2}+\left(y-y^{\prime}\right)^{2}+\left(z-z^{\prime}\right)^{2}}{4 \alpha t}\right)
$$

The temperature field in the sample due to an energy density distribution $Q\left(\mathrm{~J} \cdot \mathrm{m}^{-3}\right)$ in $t=0$ is then given by Eq. (2) where $r=(x, y, z)$.

$$
T(r, t)=\int_{\text {sample }} Q\left(r^{\prime}\right) T_{\text {Dirac }}\left(r-r^{\prime}, t\right) d r^{\prime}
$$

The artifice used for the "Dirac algorithm" is to discretize the 3D-sample and to consider each point of discretisation as a point-like source, with an energy density $Q\left(r^{\prime}\right)=Q_{0} \eta\left(r^{\prime}\right)$. Here $Q_{0}$ is the maximum value of the energy density and $\eta\left(r^{\prime}\right)$ represents the normalized energy density distribution ranging between 0 (no heat source) and 1 (full heat source). Since the infrared camera can only measure the temperature at the surface of the sample, the problem can be written in an operator form as given by Eq. (3) where each $T^{(i)}$ is a vector that contains the $2 \mathrm{D}$ temperature distribution at the sample's surface at time $t_{i}$.

$$
\left(\begin{array}{c}
A^{(1)} \\
A^{(2)} \\
\vdots \\
A^{(n)}
\end{array}\right) Q_{0}[\eta]=\left[\begin{array}{c}
T^{(1)} \\
T^{(2)} \\
\vdots \\
T^{(n)}
\end{array}\right] \Leftrightarrow \mathbf{A} Q_{0} \eta=T
$$

$\mathbf{A}$ is a matrix of size $n_{c} \cdot n_{t} \times n_{s}$ and $T$ a vector of size $n_{c} \cdot n_{t}$, where $n_{c}, n_{t}, n_{s}$ are respectively the number of discretisation of the surface's sample (detector plane of the camera), of time and of the volume that contains the sources respectively. Eq. (3) represents a linear problem of type $\mathbf{A} x=b$ where the objective is to retrieve the unknown $x$ (here the data $\eta$ ) with an inversion method.

However, in practice, all surface temperature obtained by the camera are affected by noise, so the shape of the source can only be retrieved from noisy data, $T_{\delta}^{(i)}$. Noisy data can be considered as the sum of exact data $T^{(i)}$, and a certain amount of noise being $\delta$ the noise level [4], defined by Eq. (4), where $\|\cdot\|_{2}$ represents the norm 2.

$$
\delta^{2}=\sum_{i=1}^{i_{\max }}\left\|T_{\delta}^{(i)}-T^{(i)}\right\|_{2}^{2}
$$

Due to the presence of noise, Eq. (3) is not valid anymore. It becomes an approximation, and the inversion must then be considered as a minimization problem.

\subsection{Algorithm of the inverse problem}

The algorithm for the inverse problem has been computed in two steps, first without noise (theoretical problem) and then with noise (real problem). Working on the inverse problem without noise first enables to understand the importance and role of each parameter during the inversion procedure, and therefore to better apprehend the real problem (with noise).

\subsubsection{Inversion algorithm without noise}

If there is no noise, the problem that must be inversed is then given by Eq. (5).

$$
\mathbf{A} \eta=T
$$

In practice, $\mathbf{A}$ is not square. Its row's number is largely higher than its column. Therefore, $\mathbf{A}$ is not invertible. The algorithm used for our problem is based on the Moore-Penrose pseudoinverse method [5-6]. For any matrix A, its pseudoinverse exists, is unique, and has the same dimension as $\mathbf{A}^{T}$. To form the pseudoinverse of $\mathbf{A}$, the Singular Value Decomposition (SVD) is used. In the SVD, the $m$ by $n$ matrix $\mathbf{A}$ is decomposed in 3 matrices as in Eq. (6),

$$
\mathbf{A}=\mathbf{U S V}^{T}
$$


where $\mathbf{U}$ is an orthogonal $m$ by $m$ matrix, $\mathbf{V}$ an orthogonal $n$ by $n$ matrix and $\mathbf{S}$ an $m$ by $n$ "diagonal matrix". The diagonal elements of $\mathbf{S}$ are called singular values, are positives and are arranged in decreasing size such as $s_{1} \geq s_{2} \geq \cdots \geq s_{n} \geq 0$ [4]. Since $\mathbf{U}, \mathbf{V}$ are orthogonal, $\mathbf{U}^{-1}=\mathbf{U}^{T}, \mathbf{V}^{-1}=\mathbf{V}^{T}$.

If only the first $p$ singular values are different from zero $(p<m)$, we can reduce the problem and Eq. (5) becomes

$$
\mathbf{A}=\mathbf{U}_{\mathbf{p}} \mathbf{S}_{\mathbf{p}} \mathbf{V}_{\mathbf{p}}^{\mathbf{T}}
$$

and then the inversion of Eq. (6) gives the final result, given by Eq. (8).

$$
\eta=\mathbf{V}_{\mathbf{p}} \mathbf{S}_{\mathbf{p}}^{-1} \mathbf{U}_{\mathbf{p}}^{\mathbf{T}} T
$$

As $\mathbf{S}_{p}^{-1}$ is diagonal with strictly positives parameters, Eq. (8) can be written as [4]

$$
\eta=\sum_{k=1}^{p} \frac{\mathbf{U}_{-, \mathrm{k}}^{\mathrm{T}} T}{s_{k}} \mathbf{V}_{., \mathrm{k}}
$$

Different examples have been tested to validate this method. An single point-like heat source at different depths, two points-like heat sources separated along a direction but at the same depth, and two point-like heat sources along the same line, but at different depths, i.e. one source above the other.

The example that will be presented in this paper is a combination of all these cases and is illustrated by figure 2 . Three heat sources (numbered from 1 to 3 ) are distributed on the same vertical plane $\pi(x=0)$. Each source is considered as a spatial point source with Dirac excitation. The aim is to retrieve the heat sources situated on plane $\pi$ from the transient front face temperature fields measured on the surface of the sample $\left(Z_{0}\right)$.

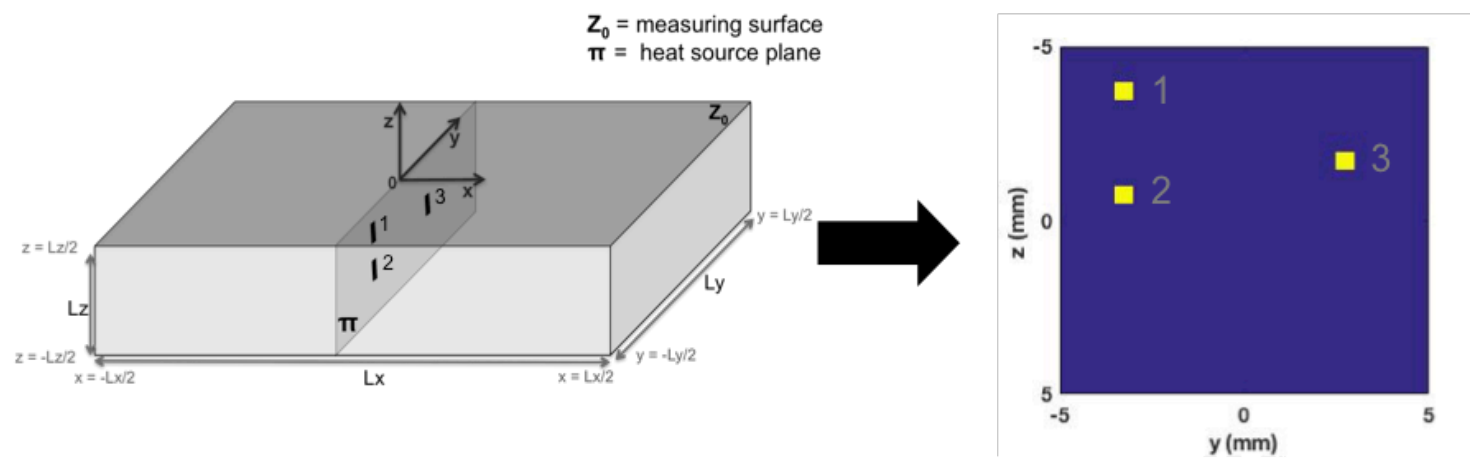

Fig. 2: Geometry of the problem and distribution of buried heat sources contained in plane $\pi$.

The material is considered isotropic. The measurements of temperature from the camera are taken between $t=0$ (time of the Dirac excitation) and $t=t_{c}$, where $t_{c}=(L z)^{2} / 4 \alpha$ is the characteristic time of diffusion. This lapse of time is then equally discretized in $n_{t} \cdot \Delta t$. Therefore, $n_{t}$ measurements are available corresponding to the temperatures distributions at each time $t_{i} \in\left[0, t_{c}\right]$.

Several reconstructions have been obtained using different temporal discretizations. Figure 3 gives the reconstructed source distribution for three different time discretizations: $n_{t}=10, n_{t}=100, n_{t}=500$. This study has been made for a spatial discretization of 64 by 64 at the surface (detector plane of the camera) and 20 by 20 for the source's plane, $\alpha=4 \mathrm{~mm}^{2} . \mathrm{s}^{-1}, \rho=1170 \mathrm{~kg} \cdot \mathrm{m}^{-3}, c_{p}=1000 \mathrm{~J} \cdot \mathrm{m}^{3} \cdot \mathrm{K}^{-1}$. To simplify the problem, we take as hypothesis $Q_{0}=1$.

With only 10 steps of time, the sources are badly reconstructed. The sources 1 and 2 are confounded and even if the location of the source 3 is quite accurate, its intensity $(0.18)$ is far from the intensity of the real source (1). However, the bigger $n_{t}$ is, the better the reconstruction is accurate. Indeed, with a discretization of 500 the three sources are almost perfectly reconstructed. 
(a) : $t_{c} / 10$

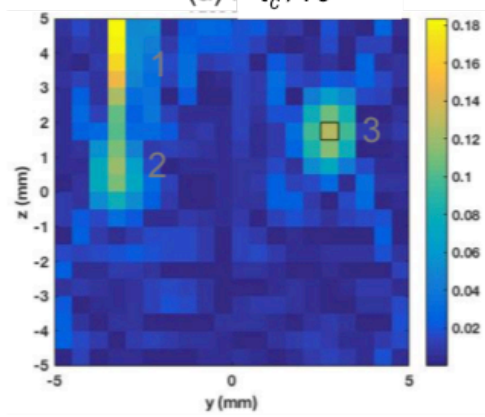

(b) : $t_{c} / 100$

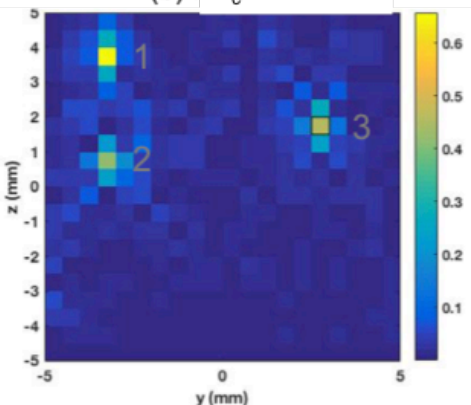

(c) : $t_{c} / 500$

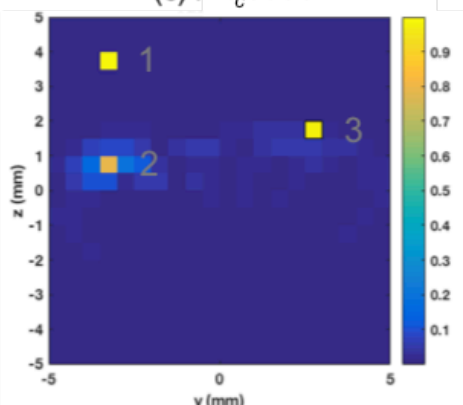

Fig. 3: Heat sources reconstruction (intensity, position) with increasing number of time steps (10, 100 and 500)

A second interesting result is that with this "Dirac method", it seems possible to reconstruct two heat sources situated one above the other, as the sources 1 and 2 are in figure 2 and 3.

To see the influence of the number of time discretization on the inversion, figure 4 draws the intensity of source 3 along the $y$ and $z$ directions of the plane for the three different number of $n_{t}(10,100$ and 500$)$.
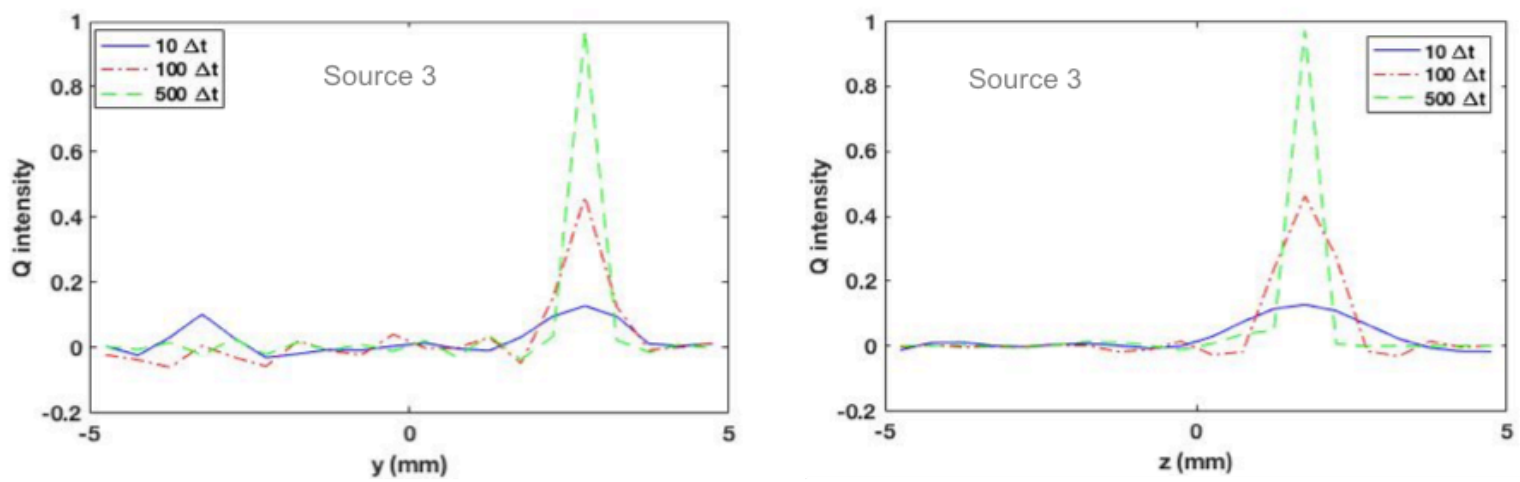

Fig. 4: Intensity of the reconstructed source 3 along the directions y (left) and z(right).

The intensity of the source converge indeed to 1 when the number of time discretizations increases. However, the more the steps number increases, the larger the matrix $\mathbf{A}$ is, and the more the computation of the inversion algorithm takes time. Figure 5 enables to see the time computation of the SVD for different number of steps time. Indeed, SVD is the more time-consuming step in the inversion algorithm, other calculations are only product of matrices.

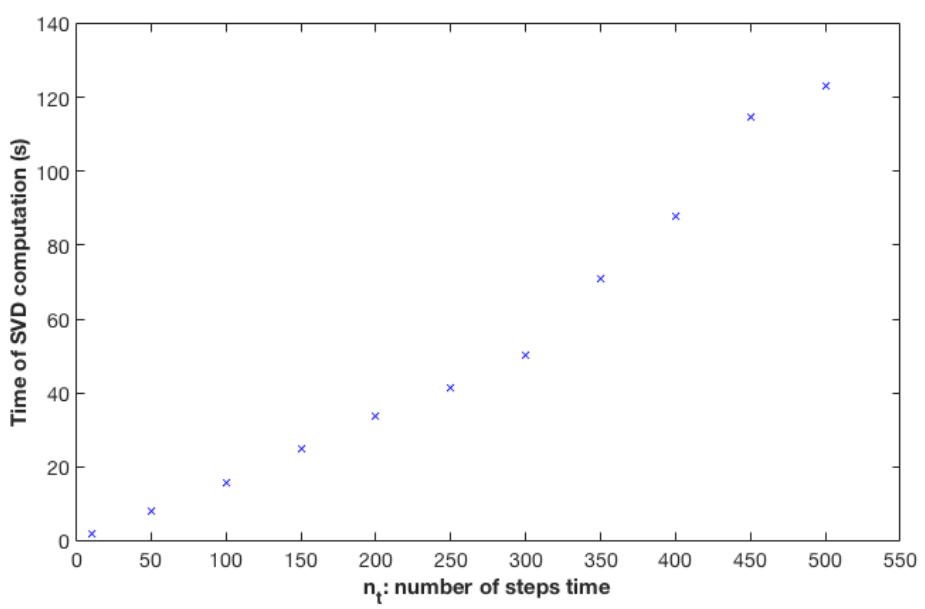

Fig. 5: Time of computation of the SVD as a function of the number of time steps (discretization of 64 by 64 at the surface and 20 by 20 for the source's plane). 
For lower number of steps times $(<300)$, the time of SVD computation seems to increase linearly with the number of time steps, however that is not the case anymore for higher discretizations. It's also important to note the spatial discretization chosen for this study is really coarse. In practice, the spatial discretization is much more extensive, then computation of the inversion algorithm is much more time-consuming.

\subsubsection{Inversion algorithm with noise}

In practice, the temperature distribution is always noised. Therefore, the problem that must be inversed is then given by Eq. (10)

$$
\mathbf{A} \eta \approx T_{\delta}
$$

The computation of the SVD for the noised problem is exactly the same as with the theoretical problem, because only the temperature distribution is impacted by the noise. However, as in [3], Eq. (2) is a system of Fredholm's integral equations of the first kind with a smooth kernel $T_{\text {Dirac }}\left(r-r^{\prime}, t\right)$. This kind of integral equations gives rise to severe ill-posedness of the inverse problem [7]. Therefore the inverse procedure needs regularization. In our inverse problem, the regularization carried out is based on the zero-order Tikhonov regularization [8]. Eq. (9) with Tikhonov regularization is modified and becomes Eq. (11) where $\alpha_{T k v}$ is called the Tikhonov parameter.

$$
\eta=\sum_{k=1}^{p} \frac{s_{k}^{2}}{s_{k}^{2}+\alpha_{T k v}} \frac{\mathbf{U}_{., \mathbf{k}}^{\mathbf{T}} T_{\delta}}{s_{k}} \mathbf{V}_{., \mathbf{k}}
$$

As it is, this regularisation can be seen as a factor on the singular values of the SVD. The choice of the Tikhonov's parameter depends principally on the noise level [4]. To see the influence of this factor, figure 6 compares the logarithm of the inverse of the singular values with and without the regularization factor, here taken (almost) arbitrarily as $\alpha_{T k v}=0.0102$. The number of time steps taken is here $n_{t}=300$.

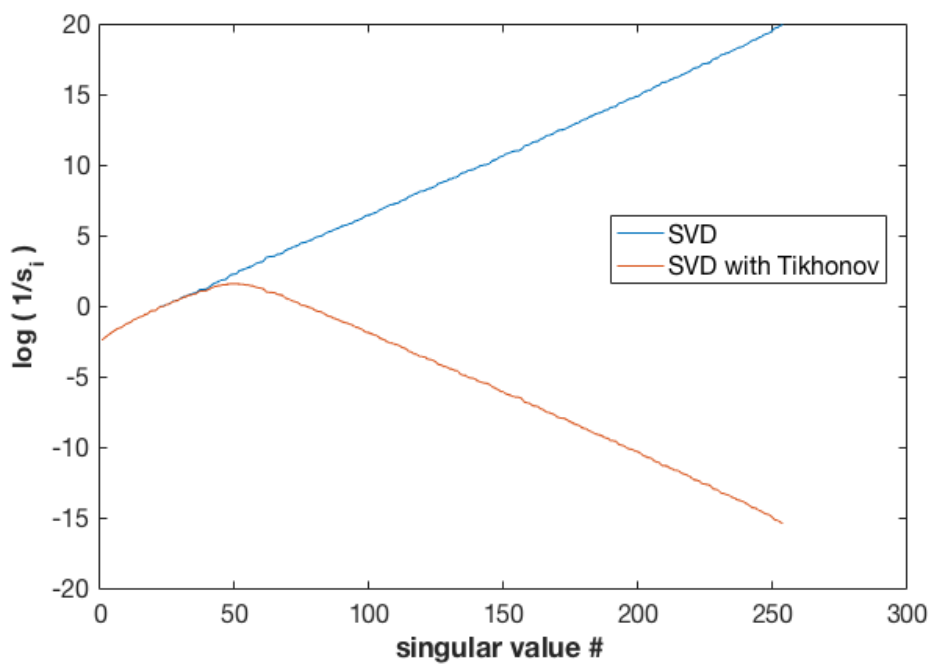

Fig. 6: Comparison between "original" and "regularized" singular values with $\alpha_{T k v}=0.0102$

From a certain threshold, the singular values become too small compared to noise level, and then the solution of the inverse algorithm diverges completely. To solve this effect, the Tikhonov regularization strongly damps the singular values and therefore makes the smaller ones negligible.

The noise on temperature distribution is very restrictive. The noisier the measured data are, the less the reconstruction of the heat sources will be accurate. The first point of our inversion procedure is to minimise the noise level. The temperature data (with noise) can be recorded in a matrix of size $n_{c} \times n_{t}$. The computation of the SVD of this matrix gives that the first modes obtained enable to reconstruct the matrix and has also for effect of reducing considerably high frequency noise [9]. The singular values obtained by the SVD of the temperature data (with the parameter given previously) are given in Figure 7. It appears indeed that only the ten first modes are of use, as the next ones have almost the same weight that is negligible. 
This method is efficient and fast. However, even if the noise level is reduced, it is not completely suppressed. Therefore a further regularization method is needed.

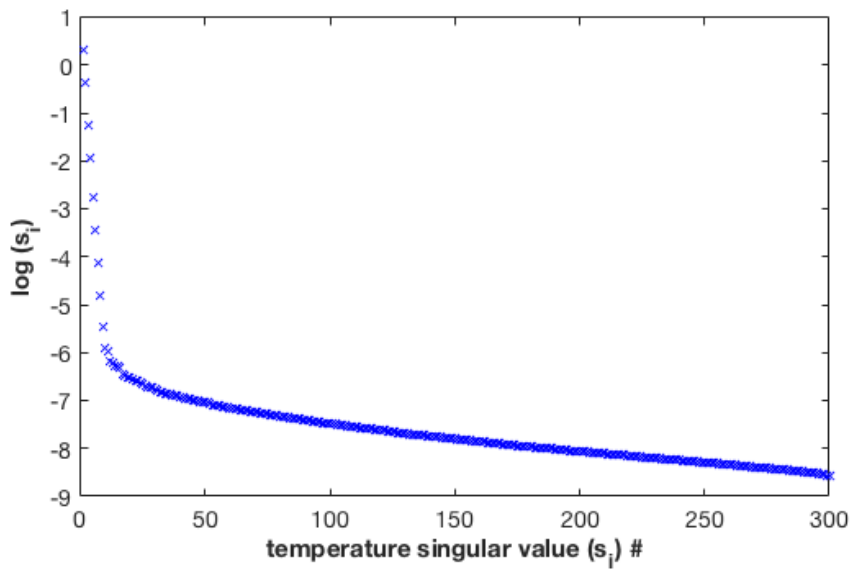

Fig. 7: Singular values of the SVD of the temperature data

Based on the results of Tikhonov regularisation, the important point is to determine the threshold from which the solution diverges. To find this limit, several reconstructions of heat sources are made. The first one is made with only one mode of the SVD (i.e. one singular value) and for each new reconstruction, the number of mode increases (with a step defined by the user of the algorithm). We stop when the number maximal of singular values are achieved. Three errors are calculated, represented by figure 8 . The first one (blue) is the error (norm 2) made between the sources reconstructed without noise (theoretical algorithm) and the real sources. The second one (red) is the error (norm 2) made between the sources reconstructed with noise without regularization and the real sources. The error decreases until 160 singular values are used, at which "time" it increases rapidly, pointing out that the solution proposed by the algorithm will diverge strongly. The threshold is then determined.

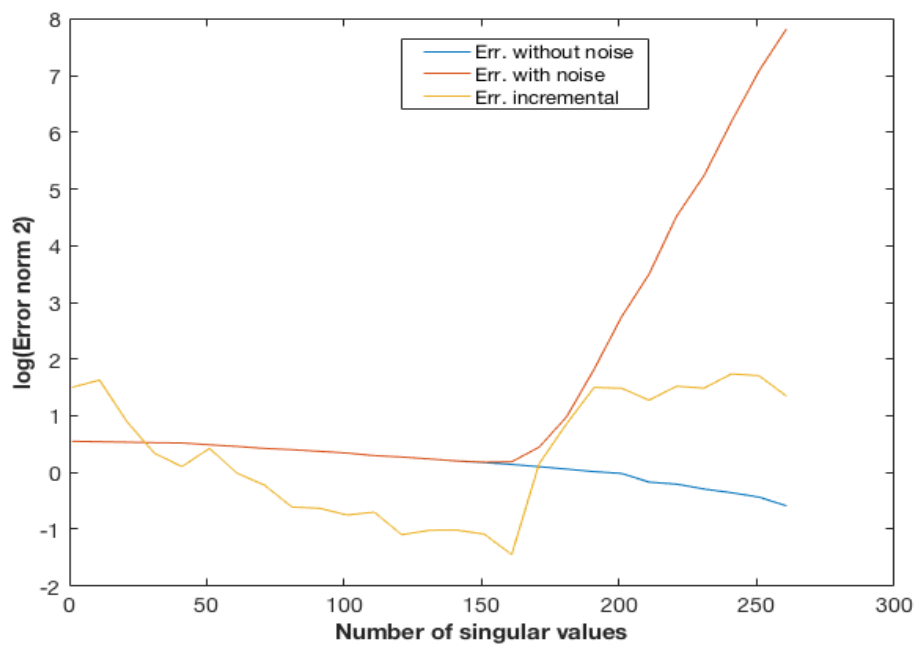

Fig. 8: Error (norm 2) made for the reconstruction of the heat sources

However, this criterion requires the knowledge of the real source. This method is then useless as the objective of the algorithm is to determine the heat sources. Therefore the last error is used (in yellow on figure 8). It represents the difference between the reconstructions of heat sources with two successive singular values numbers. For example, if the step of singular values number used between each reconstruction is 5 , then the error drawn for 100 singular values is the error made between the reconstruction with 100 singular values and these with 95 singular values. This error decreases quickly until 161, which is the threshold researched, and increases from it.

The reconstruction of the heat sources with 161 singular values is given by figure 9 . The source 1 is very well reconstructed. The sources 2 and 3 are well positioned, but their intensities are badly found. 

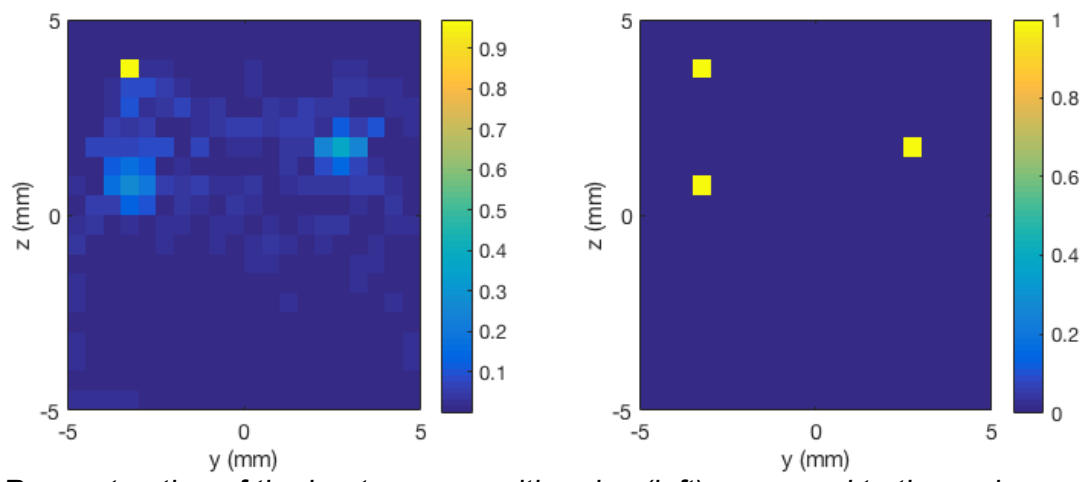

Fig. 9: Reconstruction of the heat sources with noise (left) compared to the real sources (right)

This inverse method with noise enables to reconstruct several heat sources. However, in order to retrieve the intensities of sources, the regularization method must be improved.

\section{Conclusion}

In this work, a method for characterizing buried Dirac heat source (spatial and temporal) is presented. First the theoretical method is presented it is a method that is easy to compute and gives a really good reconstruction of point sources. For the real problem (with noise) the algorithm needs regularization in order to converge to the solution. The results are promising. With this method, it is definitively possible to distinguish two heat sources when one of them is hidden below the other.

\section{REFERENCES}

[1] Mendioroz A, Celorrio R, Cifuentes A, Zaton L, Salazar A, Sizing vertical cracks using burst vibrothermography. NDT \& $E$ Int. 84, 36-46 (2016).

[2] Celorrio R, Mendioroz A, Salazar A. Characterization of vertical buried defects using lock-in vibrothermography: I. Direct problem. In Meas. Sci. Technol. 24, 065601 (2013).

[3] Celorrio R, Mendioroz A, Salazar A. Characterization of vertical buried defects using lock-in vibrothermography: II. Inverse problem. In Meas. Sci. Technol. 24, 065602 (2013).

[4] Castelo Varela A, Characterization of vertical cracks using lock-in vibrothermography, Ph.D, thesis, university of the basque country, pp 95-98, Spain (2017).

[5] Moore E. H., On the reciprocal of the general algebraic matrix, Bulletin of the American Mathematical Society, 26, 394-395 (1920)

[6] Penrose R, A generalized inverse for matrices, Mathematical Proceedings of the Cambridge Philosophical Society, 51, 406-413 (1955)

[7] Bers L, John F, Schechter M, 1964, partial Differential Equations (New York: Willey)

[8] Vogel C R, 2002, Computational Methods for Inverse Problems (Philadelphia, PA: SIAM)

[9] Bamford $M$, Méthode flash et thermographie infrarouge pour la cartographie de propriétés thermophysiques : application à la characterisation en thermomécanique, Ph.D, thesis, université de Bordeaux, pp31-37 (2007) 